\title{
Fuzzy Time Intervals for Simulating Actions
}

\author{
Vadim Ermolayev $^{1}$, Natalya Keberle ${ }^{1}$, Wolf-Ekkehard Matzke ${ }^{2}$, Richard Sohnius ${ }^{2}$ \\ ${ }^{1}$ Department of IT, Zaporozhye National University, Zhukovskogo 66, 69063, \\ Zaporozhye, Ukraine \\ vadim@ermolayev.com, kenga@zsu.zp.ua, \\ ${ }^{2}$ Cadence Design Systems, GmbH, Mozartstr. 2 D-85622 Feldkirchen, Germany \\ wolf@cadence.com, sohnius@cadence.com
}

\begin{abstract}
The paper presents time-related part of PSI ${ }^{1}$ theoretical framework. In comparison to other theories of time based on interval logics our approach presents the advancement by introducing fuzziness of time intervals as transition periods at beginnings and endings. It is argued that, though quite simple (discrete, linear, and anisotropic), our theoretical model is expressive enough to be used as a logical formalism for reasoning about stochastic, unpredictable, weakly defined action and process flows. A metric and a rich set of axiomatic relationships among time intervals are introduced for that. Further on, a means for modeling and reasoning about singular, repeated, regular events and actions having phases and vague durations is elaborated. Presented theory of time is used for modeling and reasoning about events, environmental influences, happenings, and actions while planning and scheduling in our simulations of dynamic engineering design processes.
\end{abstract}

\section{Introduction}

A major trend in engineering design today is that a design system is flexible and responsive enough to be capable of meeting and compensating sudden changes. Changing factors may be: time-to-market constraints, design specification. Unpredicted distorting external influences like sudden reorganization of a design team, increased number of design activity iterations due to the changes in the quality requirements, or factual unavailability of a required resource may also influence a design system [1]. Another important feature of a design system is the increasing geographical and cultural distribution bringing up new challenges to performance management. Indeed, provided that the parts of a design system are spread globally, the proper time management may substantially increase "round-the-clock" performance.

Time modeling is a crucial feature in a vast variety of application domains dealing with change. Probably the first to perceive it was Aristotle [2]. The importance of time modeling is shown by the numerous works in the area of temporal databases [3]

\footnotetext{
${ }^{1}$ Performance Simulation Initiative (PSI) is the project of Cadence Design Systems, GmbH.
} 
and temporal reasoning [4]. Our motivation for developing the model of time presented in this paper is the observation that accounting for mentioned specificities of changes in design processes requires a rich and expressive model of time.

The rest of the paper is structured as follows. The context and the tasks of PSI project are briefly presented in Section 2. The requirements to the model of time are devised and outlined based on the tasks of the project. Section 3 presents modeling choices based on the requirements. Section 4 describes the basic crisp part of our general model of time and time interval calculus. Section 5 introduces fuzziness in the beginnings and endings of time intervals providing means for modeling vagueness, uncertainty, and subjectivity. The means for reasoning about irregularity, regularity, and recurrence of events and actions is introduced in Section 6. Section 7 reviews the related work in the field of fuzzy temporal reasoning and compares our results to the results found in the literature. Finally concluding remarks and our plans for future work are given.

\section{Modeling Requirements}

PSI project aims at developing models, methodologies, and software tools providing rigorous engineering treatment of performance and performance management. PSI performance modeling and management approach focuses on performance as a proactive action. A fine-grained dynamic model of an Engineering Design Process is therefore developed. PSI approach considers that performance does not occur in vacuum, but is embodied in its environment and is controlled by the associated performance management process.

A Dynamic Engineering Design Process (DEDP) is a goal-directed process of transforming the representations of a design artifact in stateful nested environments. An environment comprises design artifact representations, resources, tools, and actors who perform actions to transform design artifacts using tools, consume resources. Actions are admissible in particular environmental states and may be atomic or compound, state-transitive or iterative, dependent or independent of other actions. The components of an environment may generate internal events or may be influenced by external events that are generated outside of this environment at run time. Events may have causal dependencies. A DEDP is considered a problem solving process which goals, partial goals, and environments may change dynamically. In PSI a decision taking procedure is associated with each state to allow environments adjust the course of a DEDP taking these changes into account. Decisions are taken by actors modeled by software agents.

PSI software tools are developed for assisting project managers to make robust planning, monitoring, and management of their design projects aiming at reaching best possible performance. Grounded decisions in planning are based on the knowledgebase of project logs accomplished in the past. These logs provide vast and finely grained records of the performance of the accomplished projects and may be used for simulating the behavior of the design system in response to different influences. At project execution phase PSI software may be used for predicting the behavior of the design system in the future based on the record of the partially 
accomplished DEDP, the knowledge about its environment(s), and performance simulations.

Mentioned functionalities may only be implemented if a rich and expressive model of time is used. This model should be capable of facilitating agents reasoning about environments, events, and actions employed in decision taking procedures enacted at environmental states. In particular, PSI bases its model of time on the following modeling requirements. As far as our objective in this development was providing the ontology of time for the use in agent-based software tool for DEDP simulation, the requirements were graded with respect to the use in the versions of the software as shown in Table 1. Minimal ontology of time is used in the current software of PSI software prototype. Crisp ontology of time is planned for the upcoming major version. Full ontology of time based on the fuzzy extension of the model of time will be used in the future versions. More details may be borrowed from [11].

Table 1. Required features of the model of time.

\begin{tabular}{|l|c|c|c|}
\hline \multicolumn{1}{|c|}{ Feature } & $\begin{array}{c}\text { Time } \\
\text { Minimal }\end{array}$ & $\begin{array}{c}\text { Time } \\
\text { Crisp }\end{array}$ & $\begin{array}{c}\text { Time } \\
\text { Fuzzy }\end{array}$ \\
\hline Absolute time points & $\mathrm{X}$ & $\mathrm{X}$ & $\mathrm{X}$ \\
\hline $\begin{array}{l}\text { Differently structured and grained time stamps: dates, times, time } \\
\text { zones }\end{array}$ & & $\mathrm{X}$ & $\mathrm{X}$ \\
\hline Time intervals and their durations & $\mathrm{X}$ & $\mathrm{X}$ & $\mathrm{X}$ \\
\hline Time intervals open or closed by beginning and ending instants & & $\mathrm{X}$ \\
\hline Finite and infinite time intervals & $\mathrm{x}$ & $\mathrm{X}$ \\
\hline Time intervals with vague beginnings and endings & & $\mathrm{X}$ \\
\hline Means to analyze the overlaps of time intervals & $\mathrm{X}$ & $\mathrm{X}$ \\
\hline $\begin{array}{l}\text { Subjective treatment of beginnings and endings of actions by } \\
\text { different actors }\end{array}$ & & $\mathrm{X}$ \\
\hline Sub-intervals of a time interval of an action (phases) & $\mathrm{X}$ & $\mathrm{X}$ \\
\hline $\begin{array}{l}\text { Regular intervals associated with working days, weekends and } \\
\text { irregular but repeating intervals like that associated with vacations }\end{array}$ & $\mathrm{X}$ & $\mathrm{X}$ \\
\hline $\begin{array}{l}\text { Means for modeling and analyzing changing availability, non- } \\
\text { availability, or consumption of resources with extrapolation to the } \\
\text { future }\end{array}$ & & $\mathrm{X}$ & $\mathrm{X}$ \\
\hline $\begin{array}{l}\text { Means for analyzing actor availability and occupancy in time: Who is } \\
\text { doing what, when, and what is the capacity spent rated by the overall } \\
\text { capacity? }\end{array}$ & & $\mathrm{x}$ & $\mathrm{X}$ \\
\hline $\begin{array}{l}\text { Means for modeling and analyzing instant events or events with } \\
\text { durations }\end{array}$ & & $\mathrm{x}$ & $\mathrm{X}$ \\
\hline $\begin{array}{l}\text { Means for modeling and analyzing causal relationships among events } \\
\text { in time: influences, pre-conditions, immediate-, and post-effects }\end{array}$ & & $\mathrm{X}$ & $\mathrm{X}$ \\
\hline $\begin{array}{l}\text { Means for modeling and analyzing subjective perceptions of events: } \\
\text { happenings }\end{array}$ & & $\mathrm{X}$ \\
\hline $\begin{array}{l}\text { Means for modeling and analyzing atomic and compound events } \\
\text { having different temporal parts with or without causal dependencies }\end{array}$ & $\mathrm{X}$ \\
\hline
\end{tabular}

Legend: $\mathrm{x}$ - partial coverage; $\mathrm{X}$ - full coverage dealing with vagueness and subjectivity. 


\section{Modeling Choices}

Requirements analysis revealed that a simplified model of time is appropriate for simulation purposes in PSI. Time is represented by a single time line - a linear, anisotropic, discrete set of time instants as shown in Fig. 1. It is assumed in our model of time that the following properties hold.

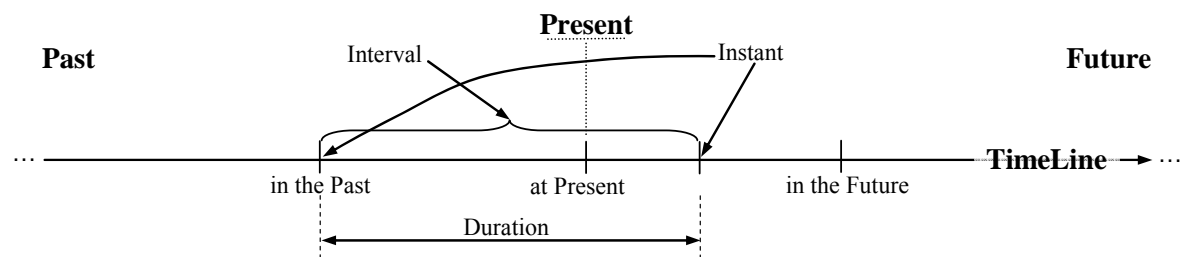

Fig. 1. An outline of the model of time.

Time is linear. Several branching time models exist. To mention just one of them, OMG adopts the model based on several time lines with different clocks and synchronization relationships for modeling and analysis of real-time and embedded systems [5]. However, for the purposes of PSI linearly anisotropic time model is sufficient. By stating that we also assume that there exists a single reference clock and all the clocks in a distributed system are synchronized with it, possibly with certain shifts representing time zones. By postulating the linearity of time we do not state that courses of action are linear. Their execution is deterministically linear in the past and at present, but may take alternative paths in the future.

Time is anisotropic. Indeed, processes are developed from the Past to the Future. If we need going back in the process we do not reverse time, but step back the subsequence of atomic actions already performed and take a repetition or a different process path. These repetitions or alternative paths are actions developed in the same temporal direction - from the Past to the Future. Events that have duration are developed in the direction from the Past to the Future. Causal relationships among Events are directed from the Past to the Future. A derivative of this assumption is that anisotropic binary relationships may be set among time instants and time intervals.

Time is discrete. Discrete model of time is used because its properties are sufficient for our simulation purposes. Computer clocks are used to measure the values of time points in simulations. These clocks have an upper bound of frequency constrained by hardware implementation. Hence, dense time, or continuous time models can not be feasibly used. The time line in PSI is used as a basic dimension in our simulation experiments, in discrete settings.

Time intervals are fuzzy. The introduction of fuzzy sets as the models of the beginnings and endings of time intervals allows accounting for uncertainty, vagueness and subjectivity in our treatment of events, happenings, actions, and their dependencies. Further on, fuzzy time intervals may be composed in interval sets denoted as periods and may contain sub-intervals denoted as phases. 


\section{Crisp Model of Time}

A Time Line is denoted as an abstract axis describing abstract time as an Abelian group $^{2}$ with respect to addition operation $(T,+)$. The elements of a Time Line are time instants (or instants in short).

An instant $t$ is a point in time having no duration. An instant has the value reflecting its position on the Time Line. The value of an instant may be:

- Negative: in this case the instant is said to be in the Past

- Zero: in this case the instant is at Present and is the Zero of the Time Line

- Positive: in this case the instant is said to be in the Future

\subsection{Linearity of Time}

Time is assumed to be linear. If put formally the linearity of time means that a total order is set among the instants on the Time Line. Given two arbitrary instants $t_{1}$ and $t_{2}$, one of the following statements holds true:

$$
\text { Before }\left(t_{1}, t_{2}\right) \text { - means that } t_{1} \text { is before } t_{2}
$$

After $\left(t_{1}, t_{2}\right)$ - means that $t_{1}$ is after $t_{2}$

Same $\left(t_{1}, t_{2}\right)$ - means that $t_{2}$ and $t_{1}$ are the same instant

The following statements on the relationships among Before, After, and Same hold true:

$$
\begin{gathered}
\text { Before }\left(t_{1}, t_{2}\right) \equiv \operatorname{After}\left(t_{2}, t_{1}\right) \\
\operatorname{Before}\left(t_{1}, t_{2}\right) \wedge \operatorname{Before}\left(t_{2}, t_{1}\right) \equiv \operatorname{Same}\left(t_{1}, t_{2}\right) \\
\operatorname{After}\left(t_{1}, t_{2}\right) \wedge \operatorname{After}\left(t_{2}, t_{1}\right) \equiv \operatorname{Same}\left(t_{1}, t_{2}\right)
\end{gathered}
$$

\subsection{Time intervals}

A time interval $I=\left[t^{b}, t^{e}\right]$ is a segment of time bounded by an instant $t^{b}$ at the beginning, an instant $t^{e}$ at the end, and Before $\left(t^{b}, t^{e}\right) \wedge \neg \operatorname{Same}\left(t^{b}, t^{e}\right)$. The latter property hints about the anisotropy of time ${ }^{3}$. From a set theoretical perspective a time interval may be denoted as an ordered triple containing its beginning instant, the set of its inner instants, and its ending instant:

$$
I=\left\{t^{b}, T^{i}, t^{e}\right\},
$$

where the order among the constituents is denoted as:

$$
\forall t_{j} \in T^{i}\left(\text { Before }\left(t^{b}, t_{j}\right) \wedge \neg \operatorname{Same}\left(t^{b}, t_{j}\right)\right) \wedge\left(\operatorname{After}\left(t^{e}, t_{j}\right) \wedge \neg \operatorname{Same}\left(t^{e}, t_{j}\right)\right)
$$

The assumption about the discrete character of time allows us to denote a unit time interval. A unit time interval is a time interval $I_{u}=\left\{t^{b}, T_{u}{ }^{i}, t^{e}\right\}$, where $T_{u}^{i}=\varnothing$. As the

\footnotetext{
${ }^{2}$ In abstract algebraic sense an Abelian group is a group in which the group operation holds commutativity.

${ }^{3}$ Otherwise we should have written $\neg \operatorname{Same}\left(t^{b}, t^{e}\right)$.
} 
set of its inner instants is empty, it comprises only two instants: the beginning instant $t^{b}$ and the ending instant $t^{e}$, therefore the following holds true:

$$
\forall t:\left(\operatorname{Before}\left(t^{b}, t\right) \vee \operatorname{After}\left(t^{e}, t\right)\right) \rightarrow\left(\operatorname{Same}\left(t, t^{b}\right) \vee \operatorname{Same}\left(t, t^{e}\right)\right)
$$

Binary relationships among time intervals are more complex than the ones over instants. Following [7], the set of binary relationships among intervals is defined below based on the binary relationships of their beginning and ending instants. Assume $I_{1}=\left[t_{1}^{b}, t_{1}^{e}\right], I_{2}=\left[t_{2}^{b}, t_{2}^{e}\right]$. Then the following hold true:

$$
\begin{aligned}
& I_{1} \text { is (distinctly) before } I_{2} \text { : } \\
& \text { Before }\left(t_{1}^{e}, t_{2}^{b}\right) \wedge \neg \operatorname{Same}\left(t_{1}^{e}, t_{2}^{b}\right) \equiv \operatorname{Before}\left(I_{1}, I_{2}\right) \\
& I_{1} \text { meets } I_{2}{ }^{4} \text { : } \\
& \text { Before }\left(t_{1}^{b}, t_{2}^{b}\right) \wedge \operatorname{Same}\left(t_{1}^{e}, t_{2}^{b}\right) \equiv \operatorname{Meets}\left(I_{1}, I_{2}\right) \\
& \text { Before }\left(t_{1}^{b}, t_{2}^{b}\right) \wedge \neg \operatorname{Same}\left(t_{1}^{b}, t_{2}^{b}\right) \wedge \text { Before }\left(t_{2}^{b}, t_{1}^{e}\right) \wedge \neg \operatorname{Same}\left(t_{2}^{b}, t_{1}^{e}\right) \\
& \wedge \text { After }\left(t_{2}^{e}, t_{1}^{e}\right) \wedge \neg \operatorname{Same}\left(t_{2}^{e}, t_{1}^{e}\right) \equiv \operatorname{Overlaps}\left(I_{1}, I_{2}\right)
\end{aligned}
$$

The relaxations of (12) result in the following:

$I_{1}$ contains $I_{2}$ :

$I_{1}$ starts $I_{2}$ :

$$
\begin{aligned}
& \text { Before }\left(t_{1}^{b}, t_{2}^{b}\right) \wedge \neg \operatorname{Same}\left(t_{1}^{b}, t_{2}^{b}\right) \wedge \operatorname{After}\left(t_{1}^{e}, t_{2}^{e}\right) \\
& \wedge \neg \operatorname{Same}\left(t_{1}^{e}, t_{2}^{e}\right) \equiv \operatorname{Contains}\left(I_{1}, I_{2}\right)
\end{aligned}
$$

$I_{1}$ finishes $I_{2}$ :

$$
\operatorname{Same}\left(t_{1}^{b}, t_{2}^{b}\right) \wedge \operatorname{After}\left(t_{2}^{e}, t_{1}^{e}\right) \wedge \neg \operatorname{Same}\left(t_{2}^{e}, t_{1}^{e}\right) \equiv \operatorname{Starts}\left(I_{1}, I_{2}\right)
$$

$I_{1}$ lies within $I_{2}$ :

$$
\operatorname{After}\left(t_{1}^{b}, t_{2}^{b}\right) \wedge \neg \operatorname{Same}\left(t_{1}^{b}, t_{2}^{b}\right) \wedge \operatorname{Same}\left(t_{1}^{e}, t_{2}^{e}\right) \equiv \operatorname{Finishes}\left(I_{1}, I_{2}\right)
$$

$$
\operatorname{Starts}\left(I_{1}, I_{2}\right) \vee \operatorname{Contains}\left(I_{2}, I_{1}\right) \vee \text { Finishes }\left(I_{1}, I_{2}\right) \equiv \operatorname{Within}\left(I_{1}, I_{2}\right)
$$

$I_{1}$ is the same as $I_{2}$ :

$$
\operatorname{Same}\left(t_{1}^{b}, t_{2}^{b}\right) \wedge \operatorname{Same}\left(t_{1}^{e}, t_{2}^{e}\right) \equiv \operatorname{Same}\left(I_{1}, I_{2}\right)
$$

\subsection{Infinite Time Intervals}

An extension of the theory of time intervals is the account for their possible infinity at the beginning, at the end, or at both ends. We shall say that a time interval ${ }^{\infty} I=\left[-\infty, t^{e}\right]=\left\{T^{i}, t^{e}\right\}$ is infinite at the beginning iff:

$$
\forall t_{j} \in T^{i} \exists t_{i}:\left(t_{i} \in T^{i}\right) \wedge \operatorname{Before}\left(t_{i}, t_{j}\right)
$$

We shall say that an interval $I^{\infty}=\left[t^{b}, \infty\right]=\left\{t^{b}, T^{i}\right\}$ is infinite at the end iff:

$$
\forall t_{j} \in T^{i} \exists t_{i}:\left(t_{i} \in T^{i}\right) \wedge \operatorname{After}\left(t_{i}, t_{j}\right)
$$

\footnotetext{
${ }^{4}$ Please note that $I_{2}$ does not meet $I_{1}$.
} 
In frame of our understanding of time there exists only one interval ${ }^{\infty} I^{\infty}=[-\infty, \infty]=\left\{T^{i}\right\}$ which is infinite at both ends - the Time Line. For ${ }^{\infty} I^{\infty}$ it holds true that:

$$
\forall t_{j} \in T^{i} \exists t_{i}, t_{k}:\left(t_{i}, t_{k} \in T^{i}\right) \wedge \operatorname{Before}\left(t_{i}, t_{j}\right) \wedge \operatorname{After}\left(t_{k}, t_{j}\right)
$$

Please notice that Past and Future on the Time Line (Fig. 1) are the intervals which are infinite respectively at the beginning and at the end and bounded by the instant of Present at their finite end:

$$
\text { Past }=[-\infty, \text { Present }]=\left\{T^{i}, \text { Present }\right\} ; \text { Future }=[\text { Present }, \infty]=\left\{\text { Present }, T^{i}\right\}
$$

Having defined Past and Future as time intervals and Present as an instant we may now reason about several temporal properties of arbitrary time intervals. We may say that an interval $I$ continues if it overlaps with the Past:

$$
\text { Overlaps }(I, \text { Past }) \equiv \text { Continues }(I)
$$

We may say that an interval $I$ ends now (at Present) if it finishes the Past (or meets the Future):

$$
\text { Finishes }(I, \text { Past }) \equiv \text { Ends }(I) \text { or Meets }(I, \text { Future }) \equiv \operatorname{Ends}(I)
$$

We may say that an interval is finished if the Past contains it:

$$
\text { Contains (Past, } I) \equiv \text { IsFinished }(I)
$$

We may say that an interval begins now (at Present) if it starts the Future (or is met by the Past):

$$
\text { Starts }(I, \text { Future }) \equiv \operatorname{Begins}(I) \text { or Meets }(\text { Past }, I) \equiv \operatorname{Begins}(I)
$$

We may say that an interval has not yet begun if it lies within the Future:

$$
\text { Contains(Future, } I) \equiv \text { NotYetBegun }(I)
$$

\subsection{Metrics of Time}

The following two aspects should be defined for associating meaningful values with the constituents of the Time Line: (i) the values of time instants; and (ii) the metric for time intervals.

Having assumed the linearity and the discreteness of Time we may denote the values of instants as mappings to integers ${ }^{5}$. We shall first define the basic mapping presuming that Present maps to zero of integers and the instants in the Past and the Future map to negative ${ }^{6}$ and positive integers respectively. We shall then refine this mapping by introducing the instant in the Past, being the beginning of measured time.

Let us associate the value equal to zero with the instant $t_{0}$ (at Present - refer to Figure 1). Then an arbitrary instant $t_{p}$ located in $n$ unit time intervals to the Past of $t_{0}$, will have the value equal to $-n$ and an arbitrary instant $t_{f}$ located in $m$ unit time intervals to the Future of $t_{0}$, will have the value equal to $m$.

5 The question about the clock units (nanoseconds, ..., centuries) of these integers is intentionally left open because it depends on the frequency of the available clock.

${ }^{6}$ Mappings to negative values may be percept as artificial because human calendars and other ways of measuring time operate with positive values. However, there are timelines which are similar to the one proposed here. For example, a logarithmic timeline starts in the past (at Big Bang time) and lasts till Present: http://en.wikipedia.org/wiki/Logarithmic timeline 
An alternative and, possibly, a more convenient way of defining instant values is to shift the zero point from the instant of Present to the instant which is commonly recognized as the zero reference point of time. For example, in Christian chronology such a point is the instant implicitly associated with the happening ${ }^{7}$ of the birth of Christ. It is topical to notice that year 0 does not exist in the chronology introduced by Dionysius Exiguus. Christian Era starts with Anno Domini (A.D.) which is year 1. A.D. is preceded by A.C. ${ }^{8}$ Christian chronology is the most widely accepted time measurement system in the World today. We shall therefore align the basic mappings of time instant values to integers with it, when convenient.

Relative and absolute positions of time intervals on the Time Line may be measured and compared by referring to their beginning or ending instants. One more metric aspect of an interval is its duration. It is straightforward to assume that the duration $|I|$ of a finite time interval $I=\left[t^{b}, t^{e}\right]$ is the quantity of different ${ }^{9}$ unit time intervals which lie within $I$ in the sense of (16). It is easy to prove that $|I|$ can be computed as:

$$
|I|=t^{e}-t^{b}
$$

\subsection{Structure of Instant Values and Durations: Date and Time}

Several finite time intervals and their durations are used in chronology. These intervals are associated with the phenomena occurring in the physical world. The following are trivial and very well known facts. They are discussed with a purpose of demonstrating the use of our means for modeling repeating and regular events (Section 6).

A year is a period associated with the event of revolution of the Earth around the Sun. A month is period which is the phase of a year and has the duration uncertainly equal to 1/12-th of the duration of a year. A day is a period associated with the revolution of the Earth around its own axis. An hour is the period with duration equal to $1 / 24$-th of the duration of a day. A minute is a Period with duration equal to $1 / 60$-th of the duration of an hour. A second is a Period with duration equal to $1 / 60$-th of the duration of a minute.

A calendar is eventually a rule system which sets the relationships among the durations of a year, a month and a day. It organizes periods within a year using the concept of a date. Gregorian calendar, being the actual dating system today, states that: a year contains 12 months; a month comprises 28 to 31 days; a year starts on the first day of the first month (called January); the total order among the days in a year is set by dates - the values associated with days; a date contains the number of a month complemented by the number of a day within the month. The number of a year is added to a date for setting up the order among the days of different years. The number of a year is calculated as a successive number starting from A.D.

\footnotetext{
${ }^{7}$ In our theory of events and happenings [11] a happening is the percept of the event of the occurrence of a real world phenomenon by an observer.

${ }^{8}$ Catholic Encyclopedia: http://www.newadvent.org/cathen/03738a.htm

${ }^{9}$ In the sense: Different $\left(I_{1}, I_{2}\right) \equiv \neg \operatorname{Same}\left(I_{1}, I_{2}\right)$.
} 


\subsection{Shifting Instant Values: Time Zones}

One of the important corollaries of the linearity of Time is that a time instant has one and only one value. Indeed, if supposed that it does not hold true, it is impossible to set the total order among the instants. An interesting question however is: how to reflect the existence of different time zones in such a theory.

Based on the assumed linearity of time we stand on the assumption that an instant measured at different locations corresponding to different time zones has the same value. However, a relative shift ${ }^{10}$ corresponding to the zone of measurement is associated with an instant. For example, the instant of the New Year 2007 has the only value (the quantity of unit time intervals passed from that instant to Present) irrespectively to the location where it has been percept. Time shifts are different at different locations.

\section{Fuzzy Extension of the Crisp Model of Time}

In reality time intervals may have fuzzy boundaries because human treatment of them is often vague and subjective. For example, when we say "I'll be back in a second", it normally (among humans) means that: (i) the action of returning back will take us quite a short time interval which duration is (desired to be) about one second; (ii) it is well possible that after one second passes we are still on our way; (iii) the probability of been back is greater than zero after 0.8 second time interval has passed and is definitely equal to one after 20 seconds.

Fuzzy time intervals may help modeling situations alike. We shall use our set theoretic definition of a time interval $(7,8)$ as the basic one and denote a fuzzy time interval as a fuzzy set with its membership function over the crisp set of time instants. Graphical representation is given in Fig. 2.

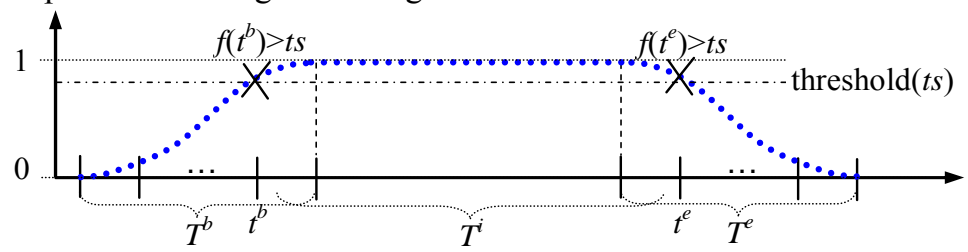

Fig. 2. A membership function of a fuzzy time interval.

A fuzzy time interval $I$ in a set theoretical sense is an ordered triple containing the fuzzy set of its beginning time instants, the (crisp) set of its inner time instants, and the fuzzy set of its ending time instants:

$$
I=\left\{T^{b}, T^{i}, T^{e}, f\right\}
$$

where:

- $T^{b}$ is the fuzzy set of instants which may be the beginning of $I$. If an instant $t_{j}^{b} \in T^{b}$ is the beginning $t^{b}$ of $I$ then $\forall t_{j}^{b}: t_{j}^{b}>t^{b} \rightarrow t_{j}^{b} \in T^{i}$

${ }^{10}$ Introduction of such a shift is natural. For example, a time zone in Italian is "fuso orario". 
- $T^{i}$ is the inner set of instants of $I$ (also called the core [12])

- $T^{e}$ is the fuzzy set of instants which may be the ending of $I$. If an instant $t_{j}^{e} \in T^{e}$ is the ending $t^{e}$ of $I$ then $\forall t_{j}^{e}: t_{j}^{e}<t^{e} \rightarrow t_{j}^{e} \in T^{i}$

- $f: Z \rightarrow[0,1]$ is a discrete membership function

The order among the constituents is denoted similarly to (8):

$$
\begin{aligned}
& \forall t_{j} \in T^{b}, t_{k} \in T^{i}, t_{l} \in T^{e}\left(\text { Before }\left(t_{j}, t_{k}\right) \wedge \neg \operatorname{Same}\left(t_{j}, t_{k}\right)\right) \\
& \wedge\left(\operatorname{After}\left(t_{l}, t_{k}\right) \wedge \neg \operatorname{Same}\left(t_{l}, t_{k}\right)\right)
\end{aligned}
$$

A considerable difficulty in setting up relationships among the elements of fuzzy time intervals and fuzzy time intervals as wholes is that their beginning and ending instants can not be crisply determined. Therefore we have to denote these relationships having in mind that any member of $T^{b}$ may appear to be $t^{b}$ and any member of $T^{e}$ may appear to be $t^{e}$. In that sense a fuzzy interval $I$ may be also denoted as $I=\left[t^{b}, t^{e}\right]-$ a segment of time bounded by an instant $t^{b}$ at the beginning, an instant $t^{e}$ at the end, and Before $\left(t^{b}, t^{e}\right) \wedge \neg \operatorname{Same}\left(t^{b}, t^{e}\right)$. In difference to a crisp interval the beginning and ending instants are located using the membership function $f$.

\subsection{Binary Relationships among Fuzzy Time Intervals}

We shall denote binary relationships (30-39) for fuzzy time intervals using (10-17) as the basics. An idea is that for fuzzy intervals their beginnings and ends are not crisp instants, but fuzzy sets of instants. Binary relationships among fuzzy intervals are more complex than the ones over intervals (10-17). The set of binary relationships among fuzzy intervals is defined below. Graphical illustration is given in Fig. 3. Assume, $I_{1}=\left\{T_{1}^{b}, T_{1}^{i}, T_{1}^{e}, f_{1}\right\}, I_{2}=\left\{T_{2}^{b}, T_{2}^{i}, T_{2}^{e}, f_{2}\right\}$. Then the following hold true:

$I_{1}$ is definitely before $I_{2}$ :

$$
\text { Before }\left(T_{1}^{e}, T_{2}^{b}\right) \equiv \text { Before }\left(I_{1}, I_{2}\right)
$$

$I_{1}$ is likely before $I_{2}$ :

$$
\forall t \in T_{1}^{e} \cap T_{2}^{b} f_{1}(t)+f_{2}(t)<1 \equiv \text { LikelyBefore }\left(I_{1}, I_{2}\right)
$$

$I_{1}$ likely meets $I_{2}$ :

$$
\forall t \in T_{1}^{e} \cap T_{2}^{b} f_{1}(t)+f_{2}(t) \geq 1 \equiv \operatorname{LikelyMeets}\left(I_{1}, I_{2}\right)
$$

$I_{1}$ definitely meets $I_{2}$ :

$$
\operatorname{Starts}\left(T_{1}^{e}, T_{2}^{i}\right) \vee \text { Finishes }\left(T_{2}^{b}, T_{1}^{i}\right) \equiv \operatorname{Meets}\left(I_{1}, I_{2}\right)
$$

$I_{1}$ overlaps $I_{2}$ :

$$
\operatorname{Meets}\left(T_{1}^{i}, T_{2}^{i}\right) \vee \operatorname{Overlaps}\left(T_{1}^{i}, T_{2}^{i}\right) \equiv \operatorname{Overlaps}\left(I_{1}, I_{2}\right)
$$

The variations of (34) result in the following:

$I_{1}$ contains $I_{2}$ :

$$
\left(\operatorname{Contains}\left(T_{1}^{i}, T_{2}^{i}\right) \vee \operatorname{Same}\left(T_{1}^{i}, T_{2}^{i}\right)\right) \wedge\left(f_{1}>f_{2}\right) \equiv \operatorname{Contains}\left(I_{1}, I_{2}\right)
$$



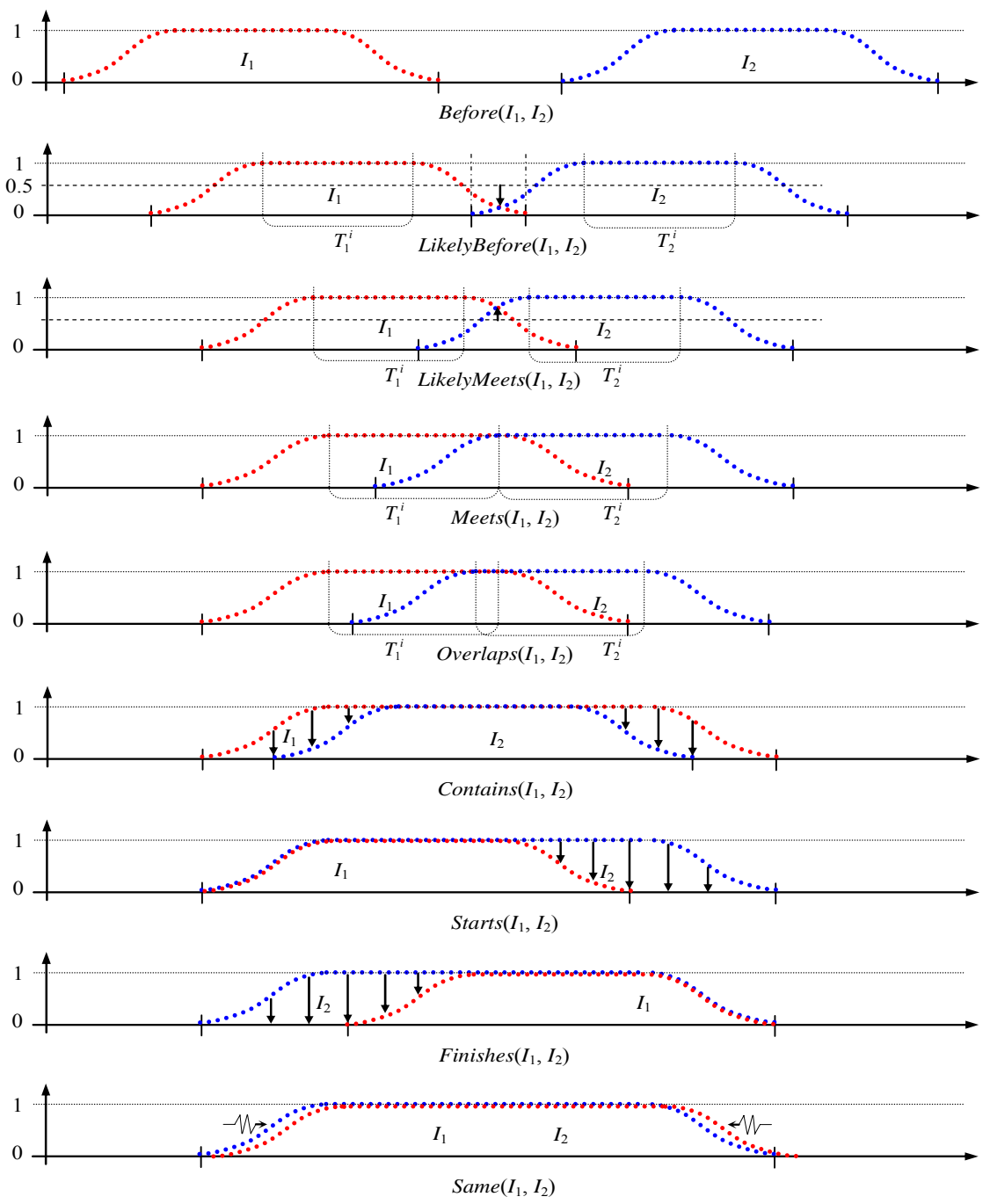

Fig. 3. Graphical representation of binary relationships among fuzzy time intervals.

$I_{1}$ starts $I_{2}$ :

$I_{1}$ finishes $I_{2}$ :

$$
\operatorname{Starts}\left(T_{1}^{i}, T_{2}^{i}\right) \equiv \operatorname{Starts}\left(I_{1}, I_{2}\right)
$$

$I_{1}$ lies within $I_{2}$ :

$$
\text { Finishes }\left(T_{1}^{i}, T_{2}^{i}\right) \equiv \text { Finishes }\left(I_{1}, I_{2}\right)
$$

$$
\operatorname{Starts}\left(I_{1}, I_{2}\right) \vee \operatorname{Contains}\left(I_{2}, I_{1}\right) \vee \operatorname{Finishes}\left(I_{1}, I_{2}\right) \equiv \operatorname{Within}\left(I_{1}, I_{2}\right)
$$

$I_{1}$ is the same as $I_{2}$ :

$$
\operatorname{Same}\left(T_{1}^{b}, T_{2}^{b}\right) \wedge \operatorname{Same}\left(T_{1}^{i}, T_{2}^{i}\right) \wedge \operatorname{Same}\left(T_{1}^{e}, T_{2}^{e}\right) \equiv \operatorname{Same}\left(I_{1}, I_{2}\right)
$$


Please note that in (30) we do not require $f_{1} \equiv f_{2}$. Therefore, $I_{1}$ and $I_{2}$ may in fact appear to be different. One of the interesting facts is that the theory of fuzzy intervals (28-39) is the proper extension of the theory of (crisp) intervals $(7,8,10-17)$.

COROLLARY: If the constituents of $I, I_{1}$, and $I_{2}$ comply with the following:

$$
\begin{gathered}
T^{b}=\left\{t^{b}\right\}, T^{e}=\left\{t^{e}\right\}, T_{1}^{b}=\left\{t_{1}^{b}\right\}, T_{1}^{e}=\left\{t_{1}^{e}\right\}, T_{2}^{b}=\left\{t_{2}^{b}\right\}, T_{2}^{e}=\left\{t_{2}^{e}\right\}, \\
\text { and } f_{1} \equiv f_{2} \equiv 1,
\end{gathered}
$$

then formulae (28-39) are logically equivalent to the formulae $(7,8,10-17)$.

OUTLINE OF THE PROOF: We shall prove the statement of the corollary by transforming each of (28-39) according to the statement and proving its logical equivalence to the corresponding formula from $(7,8,10-17)$. For example, to prove that $(7) \equiv(28)$ we shall note that if (40) are applied to (28) it is transformed as follows: $\left(I=\left\{T^{b}, T^{i}, T^{e}, f\right\}\right) \equiv\left(I=\left\{\left\{t^{b}\right\}, T^{i},\left\{t^{e}\right\}, 1\right\}\right) \equiv\left(I=\left\{t^{b}, T^{i}, t^{e}, 1\right\}\right) . f \equiv 1$ means that $t^{b}$ and $t^{e}$ always belong to $I$. From the other hand, (7) has the equivalent meaning $-t^{b}$ and $t^{e}$ always belong to $I$. Hence, formula (28) is logically equivalent to (7) under the conditions of (40). Logical equivalence in the rest is proved analogously.

Duration of a fuzzy time interval is denoted similarly to that of a crisp interval. Duration $|I|$ of a fuzzy time interval $I=\left[t^{b}, t^{e}\right]$ is the quantity of different unit time intervals ${ }^{11}$ which lie within $I$ in the sense of (38). As for crisp intervals, it is easy to prove that the duration of a fuzzy interval can be computed as:

$$
|I|=t^{e}-t^{b}
$$

\subsection{Infinite Fuzzy Time intervals}

An extension of the theory of fuzzy time intervals is the account for their possible infinity at the beginning, at the end, or at both ends. We shall say that a fuzzy time interval ${ }^{\infty} I=\left\{T^{b}, T^{i}, T^{e}, f\right\}$ is infinite at the beginning iff:

$$
T^{b}=\varnothing, \forall t_{j} \in T^{i} \exists t_{i}:\left(t_{i} \in T^{i}\right) \wedge \text { Before }\left(t_{i}, t_{j}\right)
$$

We shall say that a fuzzy time interval $I^{\infty}=\left\{T^{b}, T^{i}, T^{e}, f\right\}$ is infinite at the end iff:

$$
T^{e}=\varnothing, \forall t_{j} \in T^{i} \exists t_{i}:\left(t_{i} \in T^{i}\right) \wedge A f t e r\left(t_{i}, t_{j}\right)
$$

In frame of our model of time there exists only one interval ${ }^{\infty} I^{\infty}$ which is infinite at both ends - the Time Line. For ${ }^{\infty} I^{\infty}$ it holds true that:

$$
T^{b}=\varnothing, T^{e}=\varnothing, \forall t_{j} \in T^{i} \exists t_{i}, t_{k}:\left(t_{i}, t_{k} \in T^{i}\right) \wedge \operatorname{Before}\left(t_{i}, t_{j}\right) \wedge \operatorname{After}\left(t_{k}, t_{j}\right)
$$

11 Please note that unit time intervals are crisp intervals and their difference is denoted as Different $\left(I_{1}, I_{2}\right) \equiv \neg \operatorname{Same}\left(I_{1}, I_{2}\right)$. 


\section{Time Interval Sets: Phases and Periods}

An interval or a fuzzy interval may contain sub-intervals $(13,35)$. Sub-intervals of an interval are often associated with the phases of an event or of a process. Examples of such sub-intervals are: (i) A working week contains a working days sub-interval and a weekend. Though it seems to be crisp at a first glance, some people have five working days, but the others have six, or prefer to work on Sundays and rest on Mondays. (ii) A year contains four seasons. Though one may argue that seasons as the phases of a year and time sub-intervals of seasons within the time interval of a year are crisply defined by a calendar. However, such a crisp definition is sometimes too rigid. For example, if spring is the season when flowers blossom and grass is green, then we can not guarantee that flowers and green grass can be found in the Northern Europe on March the 1-st. From the other hand, if a calendar winter is warm enough, this may well happen in February. More details are given in [11]. (iii) A quarter contains three months. This is an example of a crisp interval containing crisp sub-intervals, at least in academic, industrial, or business settings.

A reason to consider smaller interval chunks is that an interval is associated to a phenomenon which occurs in real World and characterizes how this phenomenon is manifested in time. A phenomenon may manifest itself differently in its different phases. Phases have different sub-intervals associated to them. An evident example is: we have low temperatures and snow (so far, somewhere) in winters, but thunderstorms and high temperatures in summers. One of the examples topical to the domain of engineering design is as follows.

When a designer is assigned to perform a particular design activity he first takes his time to prepare himself for it. This preparation phase is called a Ramp-up Phase and may be spent to learn: how to use a new version of a design tool; what are the specific features of the particular design; which design techniques may be most productive in this particular case, etc. Sometimes, when designers follow trial and test approach Ramp-up and Execution time sub-intervals of these phases may overlap. They of course are better represented by fuzzy intervals than by crisp intervals.

Assume that $S=\left\{s_{1}, \ldots, s_{i}, s_{i+1}, \ldots s_{N}\right\}$ is a finite set of fuzzy time sub-intervals of a fuzzy time interval $I$, then

$$
\forall i=1 . . N-1, j=i+1,\left(\operatorname{LikelyMeets}\left(s_{i}, s_{j}\right) \vee \operatorname{Meets}\left(s_{i}, s_{j}\right)\right)
$$

and therefore

$$
\text { Same }\left(\bigcup_{i=1}^{N} s_{i}, I\right) \text {. }
$$

Please note that $(36,37)$ hold true for finite and infinite fuzzy time intervals.

Periods $(\pi)$ are fuzzy time intervals associated with regular or repeating events [11]. One may consider finite and infinite sets of periods. A finite set of periods is associated with an event having a finite number of repetitions. For example, a particular person may have not more than two periods of presidency in many countries. On the contrary, the set of year periods is infinite because the Earth will hopefully continue its revolutions around the Sun forever. 
Assume $\Pi=\left\{\pi_{1} \ldots \pi_{i}, \pi_{i+1}, \ldots, \pi_{N}\right\}$ is the finite set of periods with cardinality $N^{12}$. A total order may be set among the periods in the set:

$$
\forall i=1 . . N-1, j=i+1 . . N\left(\operatorname{Before}\left(\pi_{i}, \pi_{j}\right) \vee \operatorname{Meets}\left(\pi_{i}, \pi_{j}\right)\right)
$$

Let us denote a set of periods infinite at the beginning as ${ }^{\infty} \Pi=\left\{\ldots \pi_{i}, \pi_{i+1}, \ldots, \pi_{n}\right\}$, a set of periods infinite at the end as $\Pi^{\infty}=\left\{\pi_{1} \ldots \pi_{i}, \pi_{i+1}, \ldots\right\}$, a set of periods infinite at both ends as ${ }^{\infty} \Pi^{\infty}=\left\{\ldots \pi_{i}, \pi_{i+1}, \ldots\right\}$. Then the total ordering on these sets may be set as follows:

$$
\begin{gathered}
{ }^{\infty} \Pi^{\infty}: \forall i \exists j:\left(\operatorname{Before}\left(\pi_{i}, \pi_{j}\right) \vee \operatorname{Meets}\left(\pi_{i}, \pi_{j}\right)\right) \\
\Pi^{\infty}: \forall i \exists j>1:\left(\operatorname{Before}\left(\pi_{i}, \pi_{j}\right) \vee \operatorname{Meets}\left(\pi_{i}, \pi_{j}\right)\right) \\
{ }^{\infty} \Pi: \forall i<n \exists j:\left(\operatorname{Before}\left(\pi_{i}, \pi_{j}\right) \vee \operatorname{Meets}\left(\pi_{i}, \pi_{j}\right)\right)
\end{gathered}
$$

\section{Related Work}

There is a vast amount of published results on representing temporal information in many domains and using a broad diversity of general theories of time [13]. However, most of them are limited to crisp settings and do not deal with any form of imprecisions. Similarly to [14], we take into account such imprecisions as uncertainty, vagueness and subjectivity. Research on uncertain temporal knowledge usually studies events and associated time intervals with precise boundaries, but state that our knowledge about them is uncertain or even unknown. This category of imprecision is often modeled using possibility theory [15]. Probabilistic or possibilistic constraints on the beginnings and endings of time intervals generalizing Allen's interval temporal algebra are also introduced in $[16,17]$. Vagueness, in difference to uncertainty, means that it is impossible even to consider that an event or an action may have precise beginning or ending time instant - they have their "core" and transition periods at their ends. In this settings using probabilistic or possibilistic approaches for reasoning about the instant boundaries of an event is irrelevant. They should be substituted by time intervals as done in our work similarly to [14, 18, 19]. Speaking about subjectivity, most known approaches in temporal reasoning model subjective temporal knowledge using probability distributions expressed as fuzzy sets. However, that might be appropriate for representing uncertain subjective knowledge, but does not cope with representing subjective knowledge of vague transition periods. To the best of our knowledge, only two approaches $[14,18]$ are capable of representing subjective temporal knowledge relevantly to the requirements of PSI. The model presented in this paper is very close in its spirit to that of [14] and [18]. However, there are some differences. Both [14] and [18] use continuous temporal model - we use a discrete one. Having in mind that reasoning on fuzzy extension of Allen's temporal logic is an NP-complete problem [17], we may expect that our approach is more computationally efficient and still relevant for our project. Our approach, similarly to [18], is hybrid in the sense that both use time points and time intervals,

\footnotetext{
${ }^{12} \mathrm{~N}$ is the number of the repetitions of the phenomenon.
} 
but [14] bases on time intervals only. We consider that having time instants is important because it allows building three nested models (minimal, crisp, and full fuzzy) having more expressive ones as proper extensions of less expressive ones. For PSI it is valuable because allows increasing model expressiveness in a natural way. In difference to $[14,18]$ the model presented in this paper is more expressive because it is capable of representing sets of time intervals. This allows us reasoning about singular, repeating, and regular events [11] as well as about iterative actions and their dependencies [10]. Finally, the technique we use in implementing our ontology of time and integrating it in PSI Suite of Ontologies [8] is similar to that of [14].

\section{Concluding Remarks}

We believe that enriching such a simplistic model of time as a linear and discrete one by fuzziness of time intervals may bring substantial expressivity benefit. Accounting for the fuzziness of beginnings, meetings, durations, overlaps, and endings of concurrent or sequential actions in time may facilitate making plans and schedules of business processes more flexible and better reflecting the reality. Effective reasoning on actions in processes may make simulations of these processes more grounded. Assessments and predictions of performance based on the results of these simulations may therefore give a better match to what happens or may happen in real business settings.

The paper presented a fuzzy extension of a discrete model of time intervals. In its crisp part the model follows Allen's interval temporal calculus [7]. In its fuzzy extension our model is close to $[14,18]$, but extends them by providing means for modeling singular, repeating, and regular events [11] as well as about iterative actions and their dependencies [10]. These features are very important for our application domain. Presented model is implemented in three ontologies having different expressive power: Time Minimal, Time Crisp, and Time Full. Time Minimal is already the part of the Core of PSI Suite of Ontologies [8] and is used in the current version of PSI software prototype. Our plans for future work anticipate gradual integration of Time Crisp and Time Full ontologies in software development.

\section{Acknowledgements}

This research is performed and funded in frame of PSI project of Cadence Design Systems, GmbH. The authors would also like to acknowledge the comments of the anonymous reviewers which substantially helped in the improvement of the paper. 


\section{Bibliography}

1. Ermolayev, V., Matzke, W.-E.: Towards Industrial Strength Business Performance Management. In: Marik, V., Colombo, A.W., Vyatkin, V. (eds.) HoloMAS 2007. LNAI, vol. 4659, pp. 387-400, Springer, Heidelberg (2007)

2. Aristotle: Physics. Translated by R. P. Hardie and R. K. Gaye, eBooks@Adelaide, 2007

3. Etzion, O., Jajodia, S., Sripada, S.: Temporal Databases: Research and Practice. Springer, New York (1998)

4. Vila, L.: A survey on temporal reasoning in artificial intelligence. Artificial Intelligence Communications. 7, 4-28 (1994)

5. A UML Profile for MARTE, Beta 1. OMG Adopted Specification, http:// www.omg.org/docs/ptc/07-08-04.pdf

6. Masolo, C., Borgo, S., Gangemi, A., Guarino, N., Oltramari, A.: WonderWeb Deliverable D18. Ontology Library (final). Technical Report. Laboratory for Applied Ontology, ISTCCNR, Trento, Italy (2003)

7. Allen, J.,Ferguson, G.: Actions and Events in Interval Temporal Logic. J. of Logic and Computation, 4(5), 531-579 (1994)

8. Ermolayev, V., Jentzsch, E., Keberle, N., Sohnius, R.: Performance Simulation Initiative. The Suite of Ontologies v.2.1. Reference Specification. Technical Report PSI-ONTO-TR2007-1, VCAD EMEA Cadence Design Systems, GmbH (2007)

9. Niles, I., Pease, A.: Towards a Standard Upper Ontology. In: Guarino, N., Smith, B., Welty, C. (eds.) Proc Int. Conf. on Formal Ontologies in Information Systems (FOIS'01), pp. 2-9. ACM New York, NY, USA (2001)

10. Ermolayev, V., Jentzsch, E., Matzke, W.-E., Pěchouček, M., Sohnius, R.: Performance Simulation Initiative. Theoretical Framework v.2.0. Technical Report PSI-T-TR-2007-1, VCAD EMEA Cadence Design Systems GmbH, Feldkirchen, Germany (2007)

11. Ermolayev, V., Jentzsch, E., Keberle, N., Sohnius, R.: Performance Simulation Initiative. Theory of Time, Events, and Happenings. Technical Report PSI-T-TR-2007-2, VCAD EMEA Cadence Design Systems GmbH, Feldkirchen, Germany (2007)

12. Dubois, D., Prade, H.: Fundamentals of Fuzzy Sets. The Handbooks of Fuzzy Sets series. vol. 7, Springer (2000)

13. Schreiber, F. A.: Is Time a Real Time? An Overview of Time Ontology in Informatics. In: Halang, W. A., Stoyenko, A. D. (eds.) Real Time Computing. Springer Verlag NATO ASI, vol. F 127, pp. 283-307. Springer, Berlin (1994)

14. Nagypál, G., Motik, B.: A Fuzzy Model for Representing Uncertain, Subjective and Vague Temporal Knowledge in Ontologies. In: Meersman, R., Tari, Z., Schmidt, D. C. (eds.) ODBASE 2003. LNCS, vol. 2888, pp. 906-923. Springer, Heidelberg (2003)

15. Godo, L., Vila, L.: Possibilistic Temporal Reasoning Based on Fuzzy Temporal Constraints. In: 14th Int. Joint Conf. on Artificial Intelligence (IJCAI 95), vol.2, pp. 1916-1923. Morgan Kaufmann Publishers, Burlington, MA, USA (1995)

16. Dubois, D., Hadjali, A., Prade, H.: Fuzziness and Uncertainty in Temporal Reasoning. J. Universal Computer Science. 9(9), 1168-1194 (2003)

17. Badaloni, S., Giacomin, M.: The Algebra IAfuz: a Framework for Qualitative Fuzzy Temporal Reasoning. Artificial Intelligence. 170(10), 872-908 (2006)

18. Ohlbach, H.J.: Relations between Fuzzy Time Intervals. In: Combi, C., Ligozad, G. (eds.) 11 th Int. Symposium on Temporal Representation and Reasoning (TIME 2004), pp. 44-51. IEEE Computer Society, Washington, DC, USA (2004)

19. Schockaert, S., De Cock, M., Kerre, E. E.: Imprecise Temporal Interval relations. In: Bloch, I., Petrosino, A., Tettamanzi, A. (eds.) WILF 2005 Revised Selected Papers. LNAI, vol. 3849, pp. 108-113. Springer, Heidelberg (2006) 